Robert Fieten

Erfolgsstrategien für Zulieferer 


\section{ROBERT FIETEN}

\section{ERFOLGSSTRATEGIEN FÜR ZULIEFERER}

VON DER ABHÄNGIGKEIT ZUR PARTNERSCHAFT AUTOMOBIL- UND KOMMUNIKATIONSINDUSTRIE 


\section{Fieten, Robert:}

Erfolgsstrategien für Zulieferer : von der Abhängigkeit zur Partnerschaft, Automobil- und Informationsindustrie / Robert Fieten. - Wiesbaden : Gabler. 1991 ISBN-13: 978-3-322-82554-4

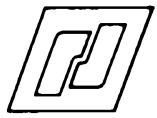

Gefördert von der Stiftung Industrieforschung. Stiftung zur Förderung der Forschung für die Gewerbliche Wirtschaft. Köln

Der Gabler Verlag ist ein Unternehmen der Verlagsgruppe Bertelsmann Intemational.

(C) Betriebswirtschaftlicher Verlag Dr. Th. Gabler GmbH, Wiesbaden 1991

Lektorat: Gudrun Böhler

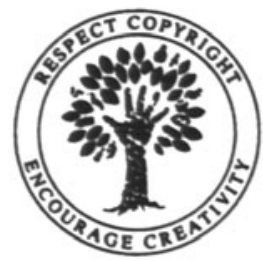

Das Werk einschließlich aller seiner Teile ist urheberrechtlich geschützt. Jede Verwertung außerhalb der engen Grenzen des Urheberrechtsgesetzes ist ohne Zustimmung des Verlages unzulässig und strafbar. Das gilt insbesondere für Vervielfältigungen, Übersetzungen. Mikroverfilmungen und die Einspeicherung und Verarbeitung in elektronischen Systemen.

Höchste inhaltliche und technische Qualität unserer Produkte ist unser Ziel. Bei der Produktion und Verbreitung unserer Bücher wollen wir die Umwelt schonen: Dieses Buch ist auf säurefreiem und chlorarm gebleichtem Papier gedruckt. Die Einschweißfolie besteht aus Polyäthylen und damit aus organischen Grundstoffen. die weder bei der Herstellung noch bei der Verbrennung Schadstoffe freisetzen.

Die Wiedergabe von Gebrauchsnamen, Handelsnamen. Warenbezeichnungen usw. in diesem Werk berechtigt auch ohne besondere Kennzeichnung nicht zu der Annahme, daß solche Namen im Sinne der Warenzeichen- und Markenschutz-Gesetzgebung als frei zu betrachten wären und daher von jedermann benutzt werden dürften.

Satzarbeiten und Graphiken: Publishing 2000, Angela Fromm. Idstein

ISBN-13: 978-3-322-82554-4 e-ISBN-13: 978-3-322-82553-7

DOI: $10.1007 / 978-3-322-82553-7$ 


\section{Vorwort}

In den Fertigungs- und Montageindustrien kommt mittelständischen Zulieferern als Partnern der großen Hersteller eine wichtige Rolle zu. Dies gilt umso mehr, als sich die Endprodukthersteller etwa in der Automobil- und Kommunikationsindustrie zunehmend auf Kernaktivitäten der Fertigung sowie Produktentwicklung und Montage konzentrieren. Gerade flexible und entwicklungsstarke Zulieferer sind daher heute unverzichtbare Kooperationspartner der großen Endprodukthersteller. Mit dem ehemaligen BDI-Präsidenten Tyll Necker läßt sich zu Recht die Frage stellen: „Was wären die Flugzeugträger der Wirtschaft ohne einen leistungsfähigen Mittelstand?“

Wenn auf der Abnehmerseite nur wenige Hersteller mit großer Kaufkraft stehen - eine für die Automobilindustrie durchaus charakteristische Situation -, so sind die Zulieferer zwangsläufig von diesen abhängig. Hierin ruht ein latentes Konfliktpotential, das dann zu Anpassungsproblemen führt, wenn die Hersteller unter stärkeren Wettbewerbsdruck geraten, in Reaktion hierauf nach neuen Formen der Arbeitsteilung mit ihren Zulieferern suchen und ihre Beschaffungs- und Logistikstrategien nachhaltig verändern. Die Zulieferer sind hiervon unmittelbar betroffen und stehen vor allem in der Automobilindustrie heute unter erheblichem Rationalisierungs- und Innovationsdruck. Sie müssen sich über umfassende Kundennutzenstrategien in einem stets anspruchsvolleren strategischen Umfeld als innovative Problemlöser neu positionieren und Anpassungsmaßnahmen in allen Teilfunktionen vornehmen.

Die vorliegende Schrift zeigt auf der Grundlage von umfangreichen Recherchen in der Automobilindustrie aber auch in der Unterhaltungselektronik als einem Teil der Kommunikationsindustrie Ansätze für die Entwicklung von offensiven Erfolgsstrategien von Zulieferern auf. Ausgehend von Michael Porters Konzept der strategischen Unternehmungsführung bietet die Untersuchung einen Einblick in die Veränderungen des strategischen Umfeldes von Automobilzulieferern, die Elemente von Erfolgsstrategien sowie die erforderlichen Veränderungen in den betrieblichen Teilfunktionen. Dabei geht es nicht darum, fertige Kochbuchrezepte für die „Strategieküche“ zu erarbeiten, vielmehr sollen betriebswirtschaftlich vorgebildeten Lesern vor allem aus der $\mathrm{Kfz}-\mathrm{Zu}$ lieferindustrie Anstöße für die strategische Neu-Positionierung ihrer Unternehmungen oder zumindest für eine kritische Standortbestimmung gegeben werden. Für Leser aus dem Bereich der Wissenschaft zeigt das Buch, wie in den letzten Jahren intensiv diskutierte Konzepte der strategischen Unternehmungsführung auf den konkreten Fall „Automobilzulieferer“ übertragen werden können.

Die vorliegende Schrift basiert zum größten Teil auf einem Forschungsprojekt, das von der Stiftung Industrieforschung (Stiftung zur Förderung der Forschung für die gewerbliche Wirtschaft), Köln finanziell gefördert wurde. Der Stiftung schulde ich großen Dank für die großzügige Unterstützung und die hervorragende Zusammenarbeit über die gesamte Laufzeit des Projektes. Ferner sei auch den vielen Interview- und Diskus- 
sionspartnern in der Zulieferindustrie gedankt, die mir bereitwillig Auskünfte über die Strategieansätze ihrer Unternehmungen gaben. Hierin einbeziehen möchte ich auch die Vertreter der Endprodukthersteller, die mir wertvolle Einblicke in ihre Beschaffungsund Logistikstrategien vermittelten. Dank gebührt darüber hinaus meinem verehrten akademischen Lehrer, Herrn Prof. Dr. Dres. h.c. mult. Erwin Grochla , unter dessen Verantwortung das Projekt akquiriert wurde, sowie Herrn Prof. Dr. Erich Frese, der mir Gelegenheit gab, das Vorhaben als Drittmittelprojekt am Seminar für Allgemeine Betriebswirtschaftslehre und Organisationslehre der Universität zu Köln durchzuführen.

Zu besonderem Dank bin ich Frau Susanne Schori sowie den Herren Robert Capek, Christoph Hahne, Dipl.-Kfm. Robert Furch und Dipl.-Kfm. Thomas Will verpflichtet, die mich in vielfältiger Weise in den einzelnen Phasen des Projektes unterstützten. Ohne die Infrastruktur der genannten studentischen Mitarbeiter wäre es mir nicht möglich gewesen, die umfangreichen Recherchen durchzuführen und die mühevolle Arbeit der Reinschrift des Manuskriptes und der Erstellung der zahlreichen Abbildungen zu bewältigen.

ROBERT FIETEN 


\section{Inhaltsverzeichnis}

Vorwort ................................. 5

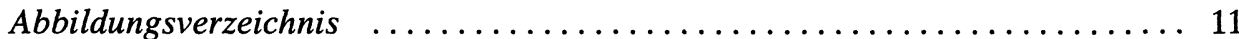

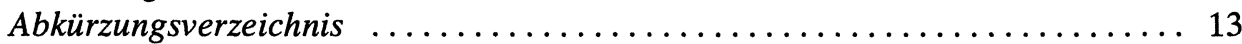

Erstes Kapitel

Zulieferer - abhängige Partner der Endprodukthersteller $\ldots \ldots \ldots \ldots \ldots 15$

1. Begriff und Merkmale der industriellen Zulieferung $\ldots \ldots \ldots \ldots \ldots \ldots \ldots$

2. Notwendigkeit einer strategischen Neu-Positionierung von Zulieferern .... 19

3. Besondere Kennzeichen der Kfz-Zulieferindustrie $\ldots \ldots \ldots \ldots \ldots \ldots \ldots 24$

4. Aufbau und empirische Grundlagen der Untersuchung $\ldots \ldots \ldots \ldots \ldots \ldots 31$

\section{Zweites Kapitel}

Strategisches Umfeld von Kfz-Zulieferern $\ldots \ldots \ldots \ldots \ldots \ldots \ldots \ldots \ldots$

1. Entwicklungstendenzen in der Weltautomobilindustrie $\ldots \ldots \ldots \ldots \ldots \ldots$

1.1 Westeuropäische Automobilindustrie $\ldots \ldots \ldots \ldots \ldots \ldots \ldots \ldots \ldots \ldots$

1.2 Nordamerikanische Automobilindustrie $\ldots \ldots \ldots \ldots \ldots \ldots \ldots \ldots 4$

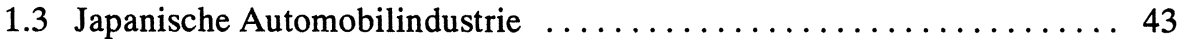

1.4 Koreanische Automobilindustrie $\ldots \ldots \ldots \ldots \ldots \ldots \ldots \ldots \ldots$

1.5 Konsequenz: Umkämpfter europäischer Markt ............ 47

2. Bestimmungsgrößen der Marktattraktivität $\ldots \ldots \ldots \ldots \ldots \ldots \ldots \ldots \ldots$

2.1 Nachfrageentwicklung und Anforderungen der Hersteller . . . . . . . . 54

2.2 Wettbewerbsumfeld und Strategien der Wettbewerber ........... 60

2.3 Beschaffungssituation und Strategien der Vorlieferanten $\ldots \ldots \ldots \ldots .63$

3. Strategische Marktsegmente im Kfz-Zuliefergeschäft $\ldots \ldots \ldots \ldots \ldots \ldots \ldots 6$ 


\section{Drittes Kapitel}

\section{Ansätze zur Entwicklung von Erfolgsstrategien}

1. Leitgedanke: Schaffung von Kundennutzen und Gewinn

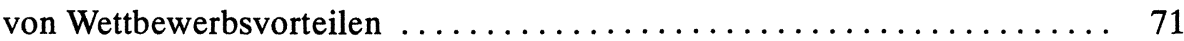

2. Management der Produktkosten und Entwicklung von Problemlösungen - Kernelemente der Strategieentwicklung . . . . . . 77

2.1 Kreatives Kostenmanagement $\ldots \ldots \ldots \ldots \ldots \ldots \ldots \ldots \ldots \ldots, 78$

2.1.1 Erfolgsfaktor dauerhaftes Produktivitätswachstum $\ldots \ldots \ldots .79$

2.1.2 Erfolgsfaktor prozeßtechnologische Neuerungen $\ldots \ldots \ldots \ldots .80$

2.2 Kreative Entwicklung von Problemlösungen $\ldots \ldots \ldots \ldots \ldots \ldots \ldots .61$

2.2.1 Erfolgsfaktor Qualität $\ldots \ldots \ldots \ldots \ldots \ldots \ldots \ldots \ldots \ldots, 82$

2.2.2 Erfolgsfaktor Flexibilität und Lieferservice $\ldots \ldots \ldots \ldots \ldots . \quad 85$

2.2.3 Erfolgsfaktor technische Entwicklung $\ldots \ldots \ldots \ldots \ldots \ldots \ldots .87$

2.2.4 Erfolgsfaktor Angebot von einbaufertigen Modulen/Teilsystemen (Integralqualität) $\ldots \ldots \ldots \ldots \ldots \ldots 89$

2.3 Strategische Marktsegmente des Zuliefergeschäftes und deren Erfolgsfaktoren $\ldots \ldots \ldots \ldots \ldots \ldots \ldots \ldots \ldots \ldots . \ldots 3$

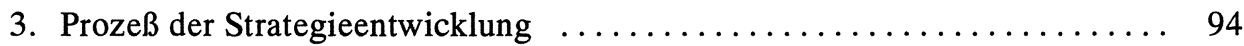

3.1 Ermittlung der strategischen Ausgangssituation $\ldots \ldots \ldots \ldots \ldots \ldots . \ldots 5$

3.2 Festlegung der strategischen Stoßrichtung $\ldots \ldots \ldots \ldots \ldots \ldots \ldots . \ldots 9$

3.3 Festlegung der Wettbewerbsstrategien ..................... 101

3.4 Festlegung der Grundsätze für die Ausgestaltung der Unternehmungsfunktionen $\ldots \ldots \ldots \ldots \ldots \ldots \ldots \ldots \ldots \ldots$

\section{Viertes Kapitel}

Vom technischen Vertrieb zum kundenorientierten Marketing $\ldots \ldots \ldots \ldots$

1. Aufgaben und Ziele des Marketings von Zulieferern $\ldots \ldots \ldots \ldots \ldots \ldots . \ldots 6$

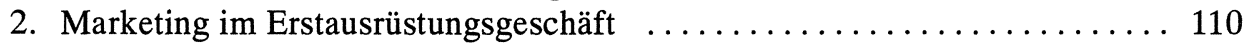

2.1 Inhaltliche Ausgestaltung des Marketings $\ldots \ldots \ldots \ldots \ldots \ldots \ldots \ldots$

2.2 Organisatorische Absicherung des Marketings durch Key-Account-Management $\ldots \ldots \ldots \ldots \ldots \ldots \ldots \ldots \ldots \ldots$

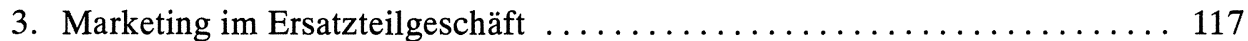

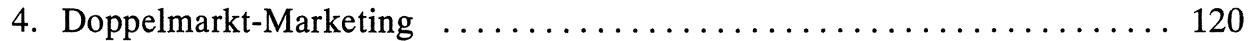

5. Wechselwirkungen zwischen Marketing und Produktentwicklung $\ldots \ldots \ldots 122$ 
Fünftes Kapitel

Von der technischen Weiterentwicklung zum

marktorientierten Forschungs- und Entwicklungsmanagement

1. Aufgaben und Ziele der Forschung und Entwicklung von Zulieferern $\ldots . .124$

2. Ausgestaltung der Forschungs- und Entwicklungsstrategie $\ldots \ldots \ldots \ldots \ldots 125$

2.1 Inhaltliche Schwerpunkte der Forschung und Entwicklung ....... 126

2.1.1 Schwerpunkte der technischen Entwicklung bei Zulieferern von Standardprodukten, Teilen und Komponenten ohne Patentschutz 126

2.1.2 Schwerpunkte der Forschung und Entwicklung bei Zulieferern von technisch anspruchsvollen Komponenten

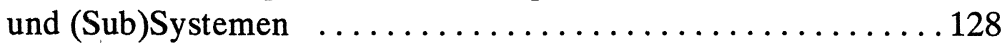

2.2 Zeitmanagement bei Forschung und Entwicklung $\ldots \ldots \ldots \ldots \ldots 131$

2.3 Technologie-Führer oder Technologie-Folger $\ldots \ldots \ldots \ldots \ldots \ldots \ldots 133$

2.4 Kooperation bei Forschung und Entwicklung $\ldots \ldots \ldots \ldots \ldots \ldots . \ldots \ldots$

\section{Sechstes Kapitel}

Von der Produktion als Vollzugsaufgabe zum strategiegerechten Produktionsmanagement

1. Leitsätze und Ziele des Produktionsmanagements von Zulieferern . ...... 140

1.1 Leitsätze des Produktionsmanagements $\ldots \ldots \ldots \ldots \ldots \ldots \ldots \ldots$

1.2 Zielsystem des Produktionsmanagements ................ 143

2. Ausgewogener Einsatz konventioneller Produktionstechnologie und moderner CA-Technologien $\ldots \ldots \ldots \ldots \ldots \ldots \ldots \ldots \ldots \ldots \ldots \ldots \ldots$

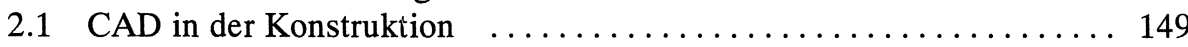

2.2 Gestaltungsansätze in Fertigung und Montage .............. 151

2.2.1 Reduzierung der Rüstzeiten durch Anpassung konventioneller Betriebsmittel ................. 152

2.2.2 Einsatz der CA-Technologien in Fertigung und Montage .... 155

2.2.3 Grenzen des Einsatzes der CA-Technologien in Fertigung und Montage ...................... 158

2.3 Organisatorische Gestaltungsparameter $\ldots \ldots \ldots \ldots \ldots \ldots \ldots \ldots \ldots$

2.3.1 Produktionsplanung und -steuerung $\ldots \ldots \ldots \ldots \ldots \ldots \ldots \ldots 16 \ldots \ldots$

2.3.2 Ansatzpunkte einer modernen Produktionsorganisation $\ldots . .169$

2.4 Personalführung und Personalentwicklung $\ldots \ldots \ldots \ldots \ldots \ldots \ldots . \ldots \ldots$

2.4.1 Wahl einer geeigneten Personalstrategie ............. 174

2.4.2 Personalführung und Personalentwicklung in den direkten Bereichen ...................... 176

2.4.3 Personalführung und Personalentwicklung in den indirekten Bereichen ..................... 177 


\section{Siebtes Kapitel}

Von der klassischen Materialwirtschaft zum Materialmanagement

1. Aufgaben und Ziele des Materialmanagements von Zulieferern ........ 182

2. Entwicklung von Beschaffungs- und Logistikstrategien $\ldots \ldots \ldots \ldots \ldots 185$

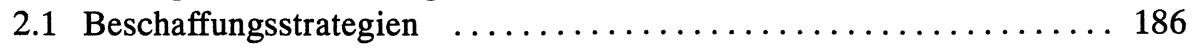

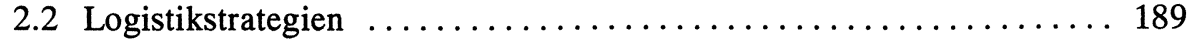

3. Organisatorische Absicherung des Materialmanagements ............ 194

3.1 Aufbauorganisatorische Gestaltungsmaßnahmen $\ldots \ldots \ldots \ldots \ldots \ldots . . \ldots 194$

3.2 Ablauforganisatorische Gestaltungsmaßnahmen .............. 196

\section{Achtes Kapitel}

Ausblick - Von der Abhängigkeit zur Partnerschaft

Literaturverzeichnis ................................ 207

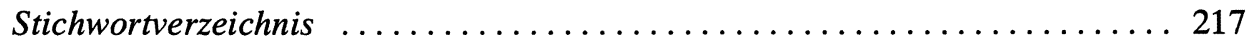




\section{Abbildungsverzeichnis}

Abbildung 1-1: Zulieferer unter Anpassungsdruck

(am Beispiel Kfz-Industrie) . ................ 22

Abbildung 1-2: Denken im strategischen Viereck - Grundlage

für die Positionierung von Zulieferern $\ldots \ldots \ldots \ldots \ldots \ldots 23$

Abbildung 1-3: Produktionsstruktur der deutschen Kfz-Teileindustrie 1988

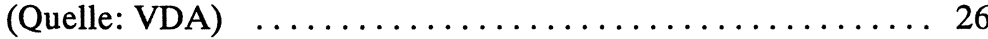

Abbildung 1-4: Produktsegmente des Kfz-Zuliefergeschäftes

(Quelle: Automobilproduktion Juni 1988) ............ 27

Abbildung 2-1: Entwicklung der Weltautomobilproduktion

(in 1000 Fahrzeugeinheiten) (Quelle: VDA)

Abbildung 2-2: Umsatz (in Mio DM) und Beschäftigte der Automobilindustrie

nach Produktionsbereichen

(Quelle: Statistisches Bundesamt, VDA)

Abbildung 2-3: Japanische Produktionskapazitäten in Westeuropa

(Quelle: BASF)

Abbildung 2-4: Marktanteile auf dem deutschen Markt für Unterhaltungs-

elektronik nach Mengen (in \%) (Quelle: GfK, Nürnberg) . . . . 48

Abbildung 2-5: Produktion, Importe und Exporte von Pkw

(in 1000 Einheiten) (Quelle: General Motors)

Abbildung 2-6: Veränderte Wettbewerbsstruktur auf dem großen europäischen

Pkw-Markt (Quelle: McKinsey, Diekmann) . ......... 51

Abbildung 2-7: Entwicklung des Marktanteils japanischer Pkw-Hersteller

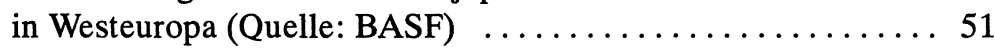

Abbildung 2-8: Strategisches Umfeld von Zulieferern $\ldots \ldots \ldots \ldots \ldots \ldots \ldots 54$

Abbildung 2-9: Anforderungen an Kfz-Zulieferer

(aus der Sicht der befragten Zulieferer) $\ldots \ldots \ldots \ldots \ldots \ldots 6$

Abbildung 2-10: Vergleich der Beschaffungsstrategien deutscher Kfz-Hersteller und eines deutschen Produzenten

von Unterhaltungselektronik ................ 57

Abbildung 2-11: Hersteller-Zulieferer-Beziehungen im Wandel . . . . . . . 59

Abbildung 2-12: Einteilung von Kfz-Zulieferern nach Ebenen (in Anlehnung

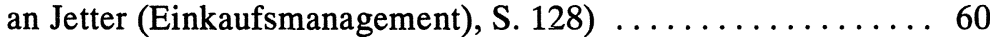

Abbildung 2-13: Begrenzter beschaffungspolitischer Spielraum

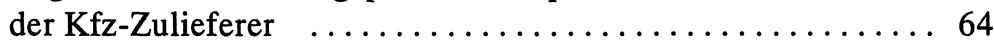

Abbildung 2-14: Strategische Marktsegmente im Zuliefergeschäft ........ 68

Abbildung 2-15: Strategische Positionierung von Zulieferern $\ldots \ldots \ldots \ldots \ldots 70$

Abbildung 3-1: Grund- und Zusatzleistungen der Zulieferer $\ldots \ldots \ldots \ldots \ldots 73$

Abbildung 3-2: Zentrale Bedeutung des Qualitätsmanagements $\ldots \ldots \ldots \ldots 74$

Abbildung 3-3: Ansatzpunkte zum Aufbau von strategischen Erfolgspotentialen

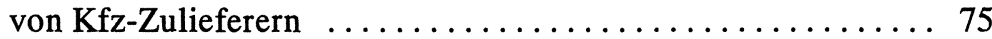


Abbildung 3-4: Strategische Marktsegmente und Erfolgsfaktoren $\ldots \ldots \ldots \ldots 93$

Abbildung 3-5: Prozeß der Strategieentwicklung ................... 94

Abbildung 3-6: Strategische Positionierungsmöglichkeiten von Geschäftsfeldern im Markt ................. 102

Abbildung 4-1: Wandel vom technischen Vertrieb zum kundenorientierten

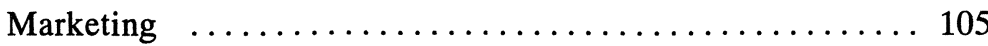

Abbildung 4-2: Marketingsituationen für Kfz-Zulieferer $\ldots \ldots \ldots \ldots \ldots \ldots . . \ldots 9$

Abbildung 4-3: Weg einer innovativen Komponente zur Serienanwendung im Automobil (Quelle: Gygax (Zuliefer-Marketing) 1988) _. 112

Abbildung 4-4: Abweichende Attraktivitätsprofile eines Zulieferproduktes aus Erstausrüster- und Endverwendersicht (Quelle: Gygax (Zuliefer- Marketing) 1988) . ......... 114

Abbildung 5-1: Abstimmung zwischen F\&E und Marketing (in Anlehnung an Brockhoff (Abstimmungsprobleme) 1985, S. 629) ...... 130

Abbildung 5-2: Chancen und Risiken für F\&E-Führer und -Folger . . ....... 134

Abbildung 5-3: Gestaltungsansätze einer Entwicklungspartnerschaft zwischen Zulieferer und Hersteller ............... 136

Abbildung 6-1: Leitsätze eines modernen Produktionsmanagements für Zulieferer . .......................... 141

Abbildung 6-2: Alternative Anpassungsstrategien zur Erreichung einer hohen logistischen (Mengen-)Flexibilität $\ldots \ldots \ldots \ldots \ldots \ldots \ldots . \ldots 147$

Abbildung 6-3: Hauptzielsetzungen von Investitionen von Zulieferern im Produktionsbereich .......................... 149

Abbildung 6-4: Wirkungskette kleinerer Losgrößen $\ldots \ldots \ldots \ldots \ldots \ldots \ldots . \ldots 154$

Abbildung 6-5: Anforderungen und Auswirkungen des Einsatzes neuer Produktionstechnologien $\ldots \ldots \ldots \ldots \ldots \ldots \ldots \ldots$. 160

Abbildung 6-6: Überblick über PPS-Konzepte $\ldots \ldots \ldots \ldots \ldots \ldots \ldots \ldots 162$

Abbildung 6-7: Abdeckung einzelner PPS-Funktionen in unterschiedlichen PPS-Konzepten (in Anlehnung an Wildemann (JIT-Konzept) 1988, S. 76) ….................. 164

Abbildung 6-8: Schlüsselqualifikationen für die Nutzung der neuen

Produktionstechnologien und der neuen Formen

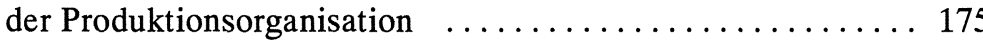

Abbildung 6-9: Einsatz neuer Produktionstechnologien als organisatorisch-personelle Herausforderung $\ldots \ldots \ldots \ldots . \ldots 179$

Abbildung 7-1: Zielsystem des Materialmanagements $\ldots \ldots \ldots \ldots \ldots \ldots . . \ldots 184$

Abbildung 7-2: Materialaufwand in Prozent des Umsatzes bei Kfz-Herstellern und -Zulieferern (Quelle: Industriekreditbank/Deutsche Industriebank) $\ldots \ldots 187$

Abbildung 7-3: Modell eines Logistikkonzeptes (in Anlehnung an Schäfer (Organisation) 1981) ...................... 190

Abbildung 7-4: Vorratsreichweiten von Kfz-Herstellern und - Zulieferern in Monaten (Quelle: Industriekreditbank/Deutsche Industriebank) 191 


\section{Abkürzungsverzeichnis}

$\begin{array}{ll}\text { Abb. } & \text { Abbildung } \\ \text { Aufl. } & \text { Auflage } \\ \text { Bd. } & \text { Band } \\ \text { BDE } & \text { Betriebsdatenerfassung } \\ \text { BE } & \text { Bauelememt } \\ \text { BMWI } & \text { Bundesministerium für Wirtschaft } \\ \text { BOA } & \text { Belastungsorientierte Auftragsfreigabe } \\ \text { bzW. } & \text { beziehungsweise } \\ \text { CA } & \text { Computer Aided } \\ \text { ca. } & \text { circa } \\ \text { CAD } & \text { Computer Aided Design } \\ \text { CAM } & \text { Computer Aided Manufacturing } \\ \text { CAP } & \text { Computer Aided Planning } \\ \text { CAQ } & \text { Computer Aided Quality Control } \\ \text { CI } & \text { Corporate Identity } \\ \text { CIM } & \text { Computer Integrated Manufacturing } \\ \text { CNC } & \text { computerized numerical controlled } \\ \text { d.h. } & \text { das heißt } \\ \text { DFÜ } & \text { Datenfernübertragung } \\ \text { DIN } & \text { Deutsche Industrie-Norm } \\ \text { DNC } & \text { distributed numerical controlled } \\ \text { DV } & \text { Datenverarbeitung } \\ \text { e.V. } & \text { eingetragener Verein } \\ \text { EG } & \text { Europäische Gemeinschaft } \\ \text { etc. } & \text { et cetera } \\ \text { F\&E } & \text { Forschung und Entwicklung } \\ \text { f. } & \text { folgende } \\ \text { ff. } & \text { fortfolgende } \\ \text { FFS } & \text { Flexibles Fertigungssystem } \\ \text { FFZ } & \text { Flexible Fertigungszelle } \\ \text { FTS } & \text { Flexibles Transportsystem } \\ \text { FZ } & \text { Fortschrittszahl } \\ \text { GfK } & \text { Gesellschaft für Kommunikationselektronik } \\ \text { hrsg. } & \text { herausgegeben } \\ \text { Hrsg. } & \text { Herausgeber } \\ \text { i. d. R. } & \text { in der Regel } \\ \text { IAA } & \text { Internationale Automobilaustellung } \\ \text { IR } & \text { Justringang } \\ \text { Jg. } & \\ \text { JIT } & \end{array}$


$\mathrm{Kfz}$

mind.

Mio.

MRP

NC

o.g.

o. V.

OES

Pkw

PPS

$\mathrm{S}$.

sog.

Sp.

u. a.

u. U.

VDA

vgl.

z. B.

z. T.
Kraftfahrzeug

mindestens

Millionen

Management Resource Planning

numerical controlled

oben genannt

ohne Verfasser

Originalersatzteil

Personenkraftwagen

Produktionsplanungs- und -steuerungs-System

Seite

sogenannte(n)

Spalte

unter anderem

unter Umständen

Verband der Automobilindustrie

vergleiche

zum Beispiel

zum Teil 